\title{
Quantum-chemical analysis of the processes at the surfaces of Diesel fuel droplets
}

\author{
S.S. Sazhin ${ }^{1 *}$, V.M. Gun'ko ${ }^{2,1}$, R. Nasiri ${ }^{1}$ \\ ${ }^{1}$ Sir Harry Ricardo Laboratories, Centre for Automotive Engineering, \\ School of Computing, Engineering and Mathematics, University of Brighton, Brighton, BN2 4GJ, UK \\ ${ }^{2}$ Chuiko Institute of Surface Chemistry, 17 General Naumov Street, Kiev 03164 Ukraine
}

\begin{abstract}
The quantum-chemical methods used for describing the processes at the surface of Diesel fuel droplets are summarised. Some results relevant to practical engineering application in Diesel engines, obtained previously, are summarised. Assuming that the droplets are so small that their interaction with individual molecules can be described using the methods of the kinetic gas theory (they can be considered as clusters/nanodrops), it was shown that the evaporation rate depends on partial pressures, temperature, and the sizes and masses of molecules and clusters/nanodrops. The results of the analysis of the collision processes between ndodecane (approximation of Diesel fuel) molecules and clusters/nanodrops, based on the dynamic reaction coordinate (DRC) method, are described. It is concluded that the probability of the attacking molecule sticking to a droplet is maximal if the molecular plane is parallel or almost parallel to the droplet surface. If the kinetic energy of the attacking molecules is high (greater than that corresponding to the boiling temperature) then it is expected that it will scatter and be removed from the cluster/nanodrop surface. The mechanisms of evaporation of microdrops and nanodrops are shown to involve rather different processes. In the case of microdrops, individual $\mathrm{C}_{12}$ molecules are evaporated from their surfaces, while in the case of nanodrops they can be disintegrated into clusters and individual molecules. The decrease in the likelihood of evaporation/condensation with temperature, predicted by the quantum-chemical (QC) approach, agrees with the prediction of the classical theory based on the MD simulations of n-dodecane molecules. The results of the estimation of the evaporation/condensation coefficient of n-dodecane molecules using the transition state theory (TST), based on the QC/DFT approach and taking into account the conformerisation of ndodecane molecules, are summarised. It is shown that taking into account the QC effects leads to marginal modifications of the predicted evaporation/condensation coefficient, particularly at temperatures which are not close to the critical temperature.
\end{abstract}

Keywords:

Diesel fuel, Droplet evaporation, Gibbs free energy, Evaporation rate, Evaporation/condensation coefficient, Quantum chemical modelling 


\section{Nomenclature}

$\begin{array}{ll}b_{i j} & \text { coefficient defined by Eq. (5) } \\ E & \text { Hamiltonian eigenvalue } \\ G & \text { Gibbs free energy } \\ H & \text { Hamiltonian operator } \\ \hbar & \text { reduced Planck constant } \\ k_{B} & \text { Boltzmann constant } \\ m & \text { mass } \\ n & \text { number of molecules } \\ N & \text { number of conformers } \\ N_{e} & \text { number of electrons in the system } \\ p & \text { pressure } \\ r_{i} ; r_{j} & \text { radii of droplets or clusters } \\ \mathbf{r} & \text { position } \\ R & \text { universal gas constant } \\ t & \text { time or the duration of the process } \\ T & \text { temperature } \\ V & \text { potential energy }\end{array}$

\section{Greek symbols}

$\begin{array}{ll}\beta & \text { evaporation coefficient } \\ \gamma & \text { evaporation rate } \\ \rho & \text { density } \\ \psi & \text { wave function }\end{array}$

\section{Subscripts}

$\begin{array}{ll}\mathrm{c} & \text { critical } \\ e & \text { electron } \\ \mathrm{ev} & \text { evaporation } \\ g & \text { gas } \\ l & \text { liquid } \\ 0 & \text { initial }\end{array}$

${ }^{*}$ Corresponding author. Tel. +44(0)1273642677; fax +44(0)1273642330; e-mail S.Sazhin@brighton.ac.uk 


\section{Introduction}

The importance of the accurate modelling of the processes at the surfaces of Diesel fuel droplets is well recognised. These processes form integral parts of the processes of Diesel droplet heating and evaporation which precede the formation of the air/fuel vapour mixture and autoignition and combustion of this mixture in Diesel engines [1]. In almost all practical engineering approaches, the most basic models for these surface processes have been used. For example, it has been assumed that droplet convective heating can be adequately described by Newton's law of cooling, and Diesel fuel vapour in the vicinity of droplet surfaces has been assumed to be always saturated. The latter assumption has allowed the modellers to reduce the problem of droplet evaporation to a much simpler problem of fuel vapour diffusion from droplet surfaces to the ambient gas (see [2] for a detailed review of this approach, commonly known as hydrodynamic approach).

The limitations of the above-mentioned approach, however, have been widely recognised since the pioneering studies of these phenomena more than a century ago (see [3] for a review of early studies). It has been shown that even in the case when the processes of droplet heating and evaporation take place at high pressures, the conventional hydrodynamic approach to their modelling is no longer valid in the immediate vicinity of droplet surfaces. It has been suggested that in this region the processes of heat and mass transfer should be modelled based on the Boltzmann equations for species (kinetic approach). In a number of studies, including [4]-[9], the heating and evaporation of Diesel fuel (approximated by n-dodecane, $\mathrm{C}_{12} \mathrm{H}_{26}$ ) droplets has been analysed based on a model using a combination of the kinetic and hydrodynamic approaches. In the immediate vicinity of droplet surfaces (up to about one hundred mean molecular free paths), the vapour and ambient gas dynamics have been studied based on the Boltzmann equation or equations (kinetic region), while at larger distances the analysis has been based on the hydrodynamic equations (hydrodynamic region). One of the important limitations of the approaches described in [4]-[9] is that they were based on the assumption that Diesel fuel can be approximated by n-dodecane. A more detailed analysis of the composition of Diesel fuel has shown that it includes in the region of a hundred or more hydrocarbon components of various kinds $[2,10,11]$.

It is not feasible to take into account the contributions of all these components in the kinetic modelling. At the same time, one can see that these components can be subdivided into two main groups: alkanes and aromatics [2]. The assumption that n-dodecane can approximate alkanes is widely used (see [10, 12, 13]), while aromatics could be approximated by p-dipropylbenzene [12]. In this case it has been suggested that a more accurate approximation of Diesel fuel, compared with the one based on its approximation by ndodecane, could be its approximation by a mixture of n-dodecane and p-dipropylbenzene. The investigation 
of the kinetic effects on heating and evaporation of two-component droplets (Diesel fuel was approximated as a mixture of n-dodecane and p-dipropylbenzene) has been described in [14].

The solution to the Boltzmann equations in the kinetic region is based on the boundary conditions at the surface of the droplet and at the interface between the kinetic and hydrodynamic regions. The boundary conditions at the interface between the kinetic and hydrodynamic regions are commonly formulated as the conservation of mass, momentum and energy fluxes at this interface. However, detailed knowledge of the processes at the surface of the droplets is required to formulate the boundary condition at this region. In most cases it has been assumed that the distribution function of the evaporating molecules is Maxwellian and the values of the evaporation coefficient have been specified.

The most common approaches to estimating the value of the evaporation coefficient have been based on molecular dynamic simulations. In $[15,16]$ these simulations have been performed based on the assumption that Diesel fuel can be approximated by n-dodecane, and the structure of n-dodecane molecules has been simplified assuming that the bonds between carbon and hydrogen atoms are much stronger than those between carbon atoms, leading to the so called United Atom Model. It has been shown that the evaporation coefficient of $n$-dodecane obtained using this approach increases with increasing temperatures and its values are reasonably close to those estimated by other methods and for other substances. These values have been used in the kinetic model for n-dodecane droplet evaporation described in [9]. Moreover, in [17] it has been shown that the distribution function of molecules in the vicinity of the droplet surface can deviate from the Maxwellian distribution, but this effect has not yet been taken into account in the kinetic modelling.

The main limitation of the model discussed in [15]-[17] is that in this model the interaction between individual molecules was described within the force field (FF) methods, which simplify both inter- and inner-molecular interactions by ignoring electrons per se. The applicability of this approach is far from obvious, as the dynamics of individual molecules in the vicinity of droplet surfaces are essentially a quantum mechanical process.

The quantum mechanical (quantum-chemical (QC)) models describing the processes at and in the vicinity of Diesel fuel droplet surfaces are described in $[11,13,18,19]$. These papers, however, primarily address the quantum chemistry community. The importance of the results presented in these papers might have been overlooked by a wider engineering audience. The main objective of the present paper is to summarise the main results reported in the above-mentioned four papers, but in a format that can be easily understood by the engineering community interested in modelling the heating and evaporation of Diesel fuel droplets.

The quantum-chemical methods used in the analysis and the compositions of Diesel fuel are discussed in Sections 2 and 3, respectively. The results of the analyses, using several approximations, are described in Section 4. The main results of the paper are summarised in Section 5. 


\section{Quantum-chemical methods of the analysis}

Assuming that processes do not explicitly depend on time, the time independent Schrödinger equation for a single particle (electrons or nuclei) with potential energy $V$ can be presented in the form

$$
E \psi(\mathbf{r})=-\frac{\hbar^{2}}{2 m} \nabla^{2} \psi(\mathbf{r})+V \psi(\mathbf{r})
$$

where $E$ is the energy (Hamiltonian eigenvalue) including potential (electrons and nuclei) and kinetic (electrons) energy components, $\psi$ is the wave function (the probability amplitude for the particle to be found at position $\mathbf{r}), H=-\frac{\hbar^{2}}{2 m} \nabla^{2}+V \psi$ is the Hamiltonian operator for a single particle, $-\frac{\hbar^{2}}{2 m} \nabla^{2}$ is the kinetic operator, $m$ is the mass of the particle, $\hbar$ is the reduced Planck constant. The potential energy is determined by all particles (electrons and nuclei) in the system.

The solution to Eq. (1) in some simple cases (e.g. isolated hydrogen atom) is well known and described in standard quantum mechanics textbooks. The main difficulty emerges when Eq. (1) is applied to the case when many particles need to be analysed simultaneously, leading to the introduction of the multi-dimensional wave function:

$$
\psi\left(\mathbf{r}_{1}, \mathbf{r}_{2}, \ldots \ldots, \mathbf{r}_{\mathbf{N}}\right)
$$

An obvious simplification of the analysis of Eq. (1) in this case is based on the assumption that the analysis of dynamics of nuclei and electrons can be separated leading to the Born-Oppenheimer (adiabatic) approximation [20].

Further simplification of Eq. (1) for the electronic part of the wave function, $\psi_{e}$, could be based on the assumption that

$$
\psi_{e}\left(\mathbf{r}_{\mathbf{1}}, \mathbf{r}_{\mathbf{2}}, \ldots \ldots, \mathbf{r}_{\mathbf{N}_{\mathbf{e}}}\right)=\psi_{e 1}\left(\mathbf{r}_{\mathbf{1}}\right) \psi_{e 2}\left(\mathbf{r}_{\mathbf{2}}\right) \ldots . . \psi_{e N_{e}}\left(\mathbf{r}_{\mathbf{N}_{\mathbf{e}}}\right) .
$$

This assumption was implicitly suggested by Hartree [21] almost 90 years ago and the modelling based on this assumption became known as the Hartree method. The wave function presented above, however, does not satisfy the Pauli principle as it does not change sign under the permutation of any pair of electrons. This problem was overcome by taking a linear combination of the functions $\psi_{e i}\left(\mathbf{r}_{\mathbf{i}}\right)$ in the form of the Slater determinant [20]:

$$
\psi_{e}\left(\mathbf{r}_{1}, \mathbf{r}_{2}, \ldots \ldots, \mathbf{r}_{\mathbf{N}_{\mathbf{e}}}\right)=\frac{1}{\sqrt{N_{e} !}}\left|\begin{array}{cccc}
\psi_{e 1}\left(\mathbf{r}_{1}\right) & \psi_{e 2}\left(\mathbf{r}_{\mathbf{1}}\right) & \ldots . . & \psi_{e N_{e}}\left(\mathbf{r}_{\mathbf{1}}\right) \\
\psi_{e 1}\left(\mathbf{r}_{\mathbf{2}}\right) & \psi_{e 2}\left(\mathbf{r}_{\mathbf{2}}\right) & \ldots . . & \psi_{e N_{e}}\left(\mathbf{r}_{\mathbf{2}}\right) \\
\ldots & \ldots & \ldots & \ldots \\
\ldots & \ldots & \ldots & \ldots \\
\ldots & \ldots & \ldots & \ldots \\
\psi_{e 1}\left(\mathbf{r}_{\mathbf{N}_{\mathbf{e}}}\right) & \psi_{e 2}\left(\mathbf{r}_{\mathbf{N}_{\mathbf{e}}}\right) & \ldots . & \psi_{e N_{e}}\left(\mathbf{r}_{\mathbf{N}_{\mathbf{e}}}\right)
\end{array}\right|
$$

Factor $1 / \sqrt{N_{e}}$ ! ensures that the wave function is normalised if the components $\psi_{e i}\left(\mathbf{r}_{\mathbf{i}}\right)$ are normalised. The value of each component for a given electron is found based on the assumption that the mean field produced 
by all other electrons is known. This leads to the need for the iterative processes leading to the calculation of the self-consistent field. This method was sometimes called the self-consistent field (SCF) method, although nowadays it is commonly known as the Hartree-Fock (HF) method (see [20] for further details).

There are two strategies for application of the HF method for practical calculations [20]. In the semiempirical methods the integrals used in the HF method are estimated based on experimental data or based on a series of rules which allow us to set certain integrals to zero. In the ab initio methods an attempt is made to calculate all these integrals.

Although the Hartree-Fock method is widely used in practical computations, this method is still an approximate one and demands considerable computational effort. This led to the development of alternative approaches to the calculation of electronic systems. The technique which gained considerable ground recently is known as the Density Functional Theory (DFT) [20]. This technique is focused on the electron density $\left(\rho_{e}\right)$ rather than on the wave function $\psi_{e}$. Hence the term 'density' in the name of the theory. In this theory it is assumed that the energy of a molecule is a function of the electron density. Since the electron density is the function of position $\rho_{e}(\mathbf{r})$, this energy appears to be the function of the function, that is functional of density. This approach appears to be not only much less demanding computationally compared with the HF method, but in some cases if can lead to more accurate results compared with the latter method.

On some occasions various approximations of the energy functional in the DFT, that incorporate parts of the exact exchange from the HF theory, have been suggested. One such approach is known as B3LYP, which stands for Becke, 3-parameter, Lee-Yang-Parr, and the exchange-correlation energy functional.

Various semi-empirical quantum chemistry methods, mentioned earlier, are important for dealing with large molecules where the full Hartree-Fock method without the approximations ( $a b$ initio approach) and DFT are too expensive. In these methods a range of fitting parameters are typically used to produce the results that best agree with experimental data or with ab initio results. One of these methods is known as the PM7 method.

The parameters in the PM7 method were calibrated to obtain results consistent with experimental and $a b$ initio data for more than 9000 compounds [22, 23]. This method can be used to study properties of compounds (molecules, polymers, and solids with up to 83 chemical elements), including thermodynamic properties (heat of formation, entropy, free energy, heat capacity). It is characterised by its average unsigned error (AUE) which is close to that of B3LYP/6-31G(d) and HF/6-31G(d) [22, 23]. As a whole, it gives the best results for a large variety of organic and inorganic compounds among other semi-empirical QCMs $[22,23]$. The main differences between the classical MM/MD, semi-empirical PM7, ab initio and DFT methods $[24,26]$ are due to the way in which the contributions of electrons are taken into account. The contribution of all electrons is taken into account in ab initio and DFT with SCF; only valence electrons are considered in semi-empirical QCMs with SCF, and no electrons per se are considered in classical MM/MD methods without SCF $[24,25,26]$. The accuracy of ab initio molecular orbital (MO) computations depends 
on the atomic orbital (AO) basis set for all electrons of atoms in molecules. These computations use the fundamental constants, as well as the mass and charge of the nuclear particles from experiments or Hartree-Fock (HF) approximations [24, 27, 25]. The electronic structure of molecules is described in MO computations as linear combinations of the AOs (MOLCAO approach) within the SCF [24]. The 6-31G(d,p) basis set has been selected as a minimal appropriate basis set to analyse the evaporation of Diesel fuel droplets [11]. Note that the accuracy of the PM7 method is close to that of the ab initio and DFT methods used with the $6-31 \mathrm{G}(\mathrm{d})$ basis set $[22,23]$.

A new continuum solvation model based on the quantum mechanical charge density of a solute molecule interacting with a continuum description of a solvent was suggested in [28]. This solvation model was called SMD, where $D$ stands for density which refers to the full solute electron density (without defining partial atomic charges). The term continuum indicates that the solvent is represented as a dielectric medium with surface tension at the solute-solvent boundary. The model separates the observable solvation free energy into two main components. The first component is the bulk electrostatic contribution arising from a selfconsistent reaction field treatment that involves the solution of the nonhomogeneous Poisson equation for electrostatics. The second component was called the cavity-dispersion-solvent-structure term and referred to the contribution arising from short-range interactions between the solute and solvent molecules in the first solvation shell. The SMD model was parameterized with a training set of almost three thousand solvation data.

In the case of modelling of the transient processes, the Dynamic Reaction Coordinates (DRC) method is widely used [29]. The key concept of this method is the Dynamic Reaction Coordinate which is the path followed by all the atoms in a system assuming the conservation of energy. In contrast to conventional molecular dynamic (MD) approaches, the contributions of the processes at the electronic level are taken into account.

The models mentioned above have been implemented in a number of known programs. In our analysis we used mainly Gaussian 09, WinGAMESS 2013 R1, and MOPAC2012.

\section{Composition of Diesel fuel}

Real-life Diesel fuel, the composition of which is described in [11, 2], was used in the analysis of [11, 13]. Molar fractions of various compounds in this fuel are the following: $13.7 \%$ paraffins, $26.4 \%$ isoparaffins, 14.9 , 7.6, and 1.6\% mono-, bi- and tri-cyclic alkanes, respectively, $16.2 \%$ alkylbenzenes, $9.2 \%$ indanes and tetralines, $8.7 \%$ naphthalenes, and small amounts of other compounds. To approximate this composition, a set of representative compounds was selected, including: normal alkanes - n-octane, n-dodecane, n-didecyl, and nheptacosane; isoalkanes - 3,6,9,10-metyl-dodecane; cycloalkanes - 1-propyl-3-hexyl-cycloheptane, diethylbicycloheptane, and ethylcycloheptane cyclononane; and aromatics - 1,4-dipropylbenzene, 1,4-dipentylbenzene, 
pentylindane, 1-propylnaphthalene, di(3-ethyl-phenyl)methane, 1-propyl-(1,2,3,4-tetrahydronaphthalene), 1-pentyl-(1,2,3,4-tetrahydronaphthalene), and ethylphenanthrene.

In some cases, a simplified approximation of Diesel fuel by alkanes or even n-dodecane have been used, although the limitations of this approach are well known (e.g. [2]).

\section{Results}

\subsection{Evaporation rate}

To the best of our knowledge, the first attempt to perform a quantum chemical study of the processes during the evaporation of real-life Diesel fuel droplets was described in [11]. The analysis of that paper was focused on the evaporation from the surface of a Diesel fuel droplet into a vacuum, described by the evaporation rate determined by the equation:

$$
\gamma=\left(\frac{1}{t}\right) \ln \left(\frac{n_{\mathrm{ev}}(t)}{n_{0}}\right),
$$

where $n_{\mathrm{ev}}(t)$ is the time dependent number of molecules leaving the droplet, $n_{0}$ is the initial number of molecules, $t$ is the duration of the process. Quantitative estimation of $\gamma$ was based on the following formula, derived by Ortega et al. [30, 31]:

$$
\gamma_{i(i+j)}=b_{i j} \frac{p}{k_{B} T n_{0}} \exp \left(\frac{\Delta G_{i+j}-\Delta G_{i}-\Delta G_{j}}{k_{B} T}\right),
$$

where $\gamma_{i(i+j)}$ is the evaporation rate of the $i$ th-molecule from a cluster (or nanodrop) $i+j, b_{i j}$ is the collision rate of the $i$ th molecule with the $j$ th cluster or nanodrop, $\Delta G_{i+j}, \Delta G_{i}$, and $\Delta G_{j}$ are the Gibbs free energies of formation of the molecules (clusters/nanodrops) from monomers (molecules) at the reference pressure $p$.

To estimate $b_{i j}$ an additional assumption was made that clusters or nanodrops are so small that their interaction with molecules can be described by the kinetic gas theory. In this case, the value of $b_{i j}$ can be inferred from the following expression [30, 31, 32]:

$$
b_{i j}=\frac{1}{\sqrt{8 \pi k_{B} T}}\left(\frac{1}{m_{i}}+\frac{1}{m_{j}}\right)^{1 / 2}\left(r_{i}+r_{j}\right)^{2},
$$

where $m_{i}$ and $m_{j}$ are the masses of the $i$ th molecule and $j$ th molecule/cluster/nanodrop, $r_{i}$ and $r_{j}$ are their radii.

Although the above-mentioned assumptions are rather restrictive for practical engineering applications, they allowed the authors of [11] to clarify the underlying physics of some of the processes at the surface of the droplets. The SMD/HF or SMD/DFT with the same 6-31G(d,p) basis set were used in [11] to estimate changes in the Gibbs free energy during the transfer of a molecule from a liquid medium into a gas phase. It was shown that the evaporation rate depends on partial pressures, temperature, sizes and masses of molecules and clusters/nanodrops. Such solvents as n-dodecane, tetraline, benzene, and isopropyltoluene 
were used to analyse the effects of surroundings on the evaporation rate of the components of Diesel fuel: normal, iso and cyclic alkanes, 1-3 ring aromatics, tetralines and indanes (in the $\mathrm{C}_{12}-\mathrm{C}_{20}$ range). It was shown that compounds $\mathrm{C}_{14}-\mathrm{C}_{16}$ make the main contribution to the Diesel fuel under consideration; all cyclic organics have the $\mathrm{C}_{1}-\mathrm{C}_{6}$ aliphatic side groups. An increase in the molecular size of alkanes from n-octane to n-heptacosane or in the aromaticity of compounds resulted in a strong decrease in the values of the evaporation rate.

The processes considered in [11] were further investigated in [13]. In contrast to [11], the analysis of [13] was focused only on alkanes as the main components of Diesel fuels, and particularly on n-dodecane, the component widely used as a representative of this fuel. The analysis of [13] was based on the assumption that the system is in a state of thermodynamic equilibrium (evaporation and condensation rates are equal). The evaporation rate was shown to decrease with increasing cluster/nanodrop diameter and decreasing temperature. The relative number of evaporated molecules, however, does not depend on cluster/nanodrop diameters, and increases with increasing temperature. At certain temperatures, the clusters/nanodrops were expected to fully evaporate. The relative number of residual molecules in clusters/nanodrops for n-alkanes in the range $\mathrm{C}_{8}-\mathrm{C}_{27}$ was shown to increase with temperature and with the carbon numbers in the molecules. Thus the evaporation process of a mixture of n-alkanes was expected to lead to increased concentration of heavy n-alkanes in droplets. This result is consistent with the one inferred from classical analyses of multi-component droplet heating and evaporation (e.g. [2]).

\subsection{Interaction between molecules and clusters/nanodrops}

The analysis described so far in this section was focused on the average or integral characteristics of the processes at the surface of the droplets. In what follows, the details of the analysis of the collision processes between $\mathrm{n}$-dodecane molecules and clusters/nanodrops are described, following [13]. The analysis of that paper was based on the Dynamic Reaction Coordinate (DRC) method. The application of this method allows one to elucidate the interaction mechanism of a molecule with a cluster/nanodrop depending on the kinetic characteristics and temperature of the system. The characteristics of this mechanism refer to scattering or sticking of the molecules. In the DRC calculations, the total kinetic energy is partitioned into the kinetic energy of random thermal bond vibrations and rotations and the kinetic energy of the translational motion of the whole molecules. In [13], the DRC method was applied to study the dependence of sticking/scattering of n-dodecane molecules on their angles of attack, kinetic energy (temperature), and cluster/nanodrop size. The DRC calculations were performed for molecules interacting with a cluster ( 7 molecules) or a nanodrop (64 or 128 molecules) of n-dodecane molecules. The results are shown in Figs. 1 and 2. The information presented in these figures is similar to that presented in Figs. 5 and 7 of [13].

As one can see from Figs. 1, at large angles of attack, a molecule is absorbed by a cluster or nanodrop of relatively small size $(d=2-7 \mathrm{~nm})$ if the kinetic energy is low and the attacking molecule is not oriented 
exactly towards one of the surface molecules (but rather between neighbouring surface molecules) (see Fig. 1b). At $\Theta \approx 1^{\circ}$ (see Fig. 1a) an almost perfectly elastic collision was observed if the molecule had relatively high velocity (kinetic energy $\sim 10 \mathrm{~kJ} / \mathrm{mol}$ or larger) and was oriented directly towards one of the surface molecules. In the DRC calculations shown in Figs. 1, the kinetic energy of the molecules in the clusters or nanodrops was low and thermal vibrations and bond rotations corresponded to 300-400 K. At the same time, the kinetic energy of the attacking molecule was high (its effective temperature was in the range 500-1200 K). In Fig. 2, the results of calculations for larger systems, using the PM7 method, are shown.

Although the clusters and nanodrops shown in Figs. 1 and 2 are not particularly good approximations of fuel droplets in real-life Diesel engine conditions, the results of our analysis allowed us to identify the correct underlying physics of the droplet evaporation/condensation processes which is generally overlooked when the conventional methods of the analysis of the phenomena are applied.

Further analyses, similar to those shown in Figs. 1 and 2, allowed the authors of [13] to conclude that the probability of the attacking molecule sticking to a droplet is maximal if the molecular plane is parallel or almost parallel to the droplet surface as this corresponds to multi-point interactions of relatively long n-dodecane molecules with the droplet surface. If the kinetic energy of the attacking molecules is high (greater than that corresponding to boiling temperature) then it is expected that they will scatter and be removed from the cluster/nanodrop surface. Molecule-nanodrop interaction results (sticking or scattering) depend on the kinetic energy and orientations of the attacking and surface molecules. It was shown that the mechanisms of evaporation of microdrops and nanodrops are likely to involve rather different processes. In the case of microdrops, individual $\mathrm{C}_{12}$ molecules are evaporated from their surfaces, while in the case of nanodrops they can be disintegrated into clusters and individual molecules.

It was shown that the decrease in the likelihood of evaporation/condensation with temperature, predicted by the analysis presented above, agrees with the prediction of the classical theory based on the MD simulations of $\mathrm{n}$-dodecane molecules (see $[15,16,17]$ ). At the same time, the analysis presented in this section does not allow us to predict the evaporation coefficient, as was done in $[15,16,17])$ using the classical FF analysis. The analysis of each collision process, similar to the ones shown in Fig. 1, required a powerful PC (three four-core PCs with a chip Core i7 were used for two-month calculations of a small systems with eight n-dodecane molecules). To study these processes using DFT/DRC methods for larger systems with dozens or hundreds of the molecules, a supercomputer would be needed. The latter was used for some calculations to study the conformerisation effects for n-dodecane (95 conformers). To quantify the values of the evaporation/condensation coefficient, using the above-mentioned analysis, one would need to repeat these calculations for a wide range of the angles of attack, orientation of molecules and energies for various conformers (cf. [19]) and various conditions of clusters and nanodrops (the effects of the size of the clusters/nanodrops would need to be investigated as well). Since this does not look feasible at the moment, an alternative approach to calculating the above-mentioned evaporation/condensation coefficient, 
taking into account quantum chemical effects, is described in the next section, following [18].

\subsection{Estimation of the evaporation/condensation coefficient}

The analysis of [18] was based on the transition state theory (TST) and quantum chemical DFT methods. These were applied to several ensembles of conformers of n-dodecane molecules. There was some similarity between the approach used in [18] and the one used previously (see [1]). In contrast to the previous studies, however, in the analysis of [18] the TST was based on a QC DFT approach taking into account the conformerisation of n-dodecane molecules. As in many previous studies, n-dodecane was considered as a representative of Diesel fuel (see [2] for the analysis of the validity of this assumption).

Several approaches to the estimation of the evaporation/condensation coefficient $\beta$ were considered in [18]. It was shown that the most accurate expression for the condensation coefficient is the one averaged over the states of various conformers transferred between two phases and given by the following formula:

$$
\left\langle\beta_{V}\right\rangle=\left\{1-\left[\frac{\rho_{g}}{\rho_{l}} \exp \frac{\left\langle\Delta G_{g \rightarrow l}\right\rangle}{R T}\right]^{1 / 3}\right\} \exp \left\{-0.5\left[\left[\frac{\rho_{g}}{\rho_{l}} \exp \frac{\left\langle\Delta G_{g \rightarrow l}\right\rangle}{R T}\right]^{1 / 3}-1\right]^{-1}\right\},
$$

where $R$ is the universal gas constant, $\rho_{g(l)}$ is the gas (liquid) density, $\Delta G_{g \rightarrow l}$ is the change in the Gibbs free energy during the condensation process, subscript ${ }_{V}$ indicates that the expression for $\beta$ explicitly depends on the specific volumes, \langle\rangle indicates averaging over the states of various conformers transferred between two phases. It is assumed that the process under consideration is quasi-steady-state and the condensation coefficient predicted by Eq. (6) is equal to the evaporation coefficient.

The effects of both the conformerisation and cross-conformerisation (changes in conformer state during transfer into another phase) of n-dodecane molecules (CDM effects), which can contribute to the Gibbs free energies of evaporation and solvation, were analysed using the MSTor program [33,34] applied to n-dodecane at 300-1200 K. Ninety-five stable conformers were selected based on the changes in the Gibbs free energy from 1000 conformers generated by ConfGen [33]. The results of calculations for these conformers are shown in Table 1. The numbers referring to conformers under consideration were random and do not depend on the value of $G$. This was done to obtain a relatively random set of conformers at various $N \leq 95$ characterised by wide distributions of the values of $G$. Details of the calculations are given in the supplementary material of $[18]$.

Note that Table 1 is slightly different from the corresponding table presented in [18], where the results of calculations were presented only for 73 out of 95 conformers. This difference, however, did not lead to any difference in the conclusions (see Fig. S2 of [18]).

A comparison between the results of calculations of $\beta$ based on Expression (6) and those obtained previously is shown in Fig. 3. Some of the results presented in this figure are reproduced from Fig. 5 of [18]. As one can see from this figure, taking into account the QC effects leads to marginal modifications of 
the predicted evaporation/condensation coefficient, except at temperatures close to the critical temperature (where this modification turned out to be significant). Thus, although the analysis of the QC effects takes into account many new effects ignored in the conventional $\mathrm{FF}$ approach, the contribution of these effects to the values of the evaporation/condensation coefficient turned out to be marginal, unless temperatures close to the critical temperature were considered.

\section{Conclusions}

The quantum mechanical (quantum-chemical) methods, used for describing the processes at Diesel fuel droplet surfaces, are summarised. These methods include the Hartree-Fock (HF) method, the Density Functional Theory (DFT), the PM7 method, the solvation model SMD, and the Dynamic Reaction Coordinates (DRC) method. Diesel fuel with the following molar fractions of compounds was used in the analysis: $13.7 \%$ paraffins, $26.4 \%$ isoparaffins, $14.9,7.6$, and 1.6\% mono-, bi- and tri-cyclic alkanes, respectively, $16.2 \%$ alkylbenzenes, $9.2 \%$ indanes and tetralines, $8.7 \%$ naphthalenes, and small amounts of other compounds. On some occasions this composition was simplified to the approximation of Diesel fuel by alkanes or even n-dodecane.

Some results relevant to practical engineering applications in Diesel engines, obtained previously, are summarised. Assuming that the droplets are so small that their interaction with individual molecules can be described using the methods of the kinetic gas theory (they can be considered as clusters/nanodrops), it was shown that the evaporation rate depends on partial pressures, temperature, sizes and masses of molecules and clusters/nanodrops. Also, it was shown that compounds $\mathrm{C}_{14}-\mathrm{C}_{16}$ make the main contribution to the Diesel fuel under consideration. An increase in the molecular size of alkanes from n-octane to n-heptacosane or in the aromaticity of compounds resulted in a strong decrease in the values of the evaporation rate. The evaporation process of a mixture of n-alkanes was shown to lead to an increased concentration of heavy n-alkanes in droplets.

The results of the analysis of the collision processes between n-dodecane (approximation of Diesel fuel) molecules and clusters/nanodrops, based on the Dynamic Reaction Coordinate (DRC) method, are summarised. In the DRC calculations, the total kinetic energy is partitioned into the kinetic energy of random thermal bond vibrations and rotations and the kinetic energy of the translational motion of the whole molecules. This approach was applied to study the dependence of sticking/scattering of n-dodecane molecules on their angles of attack, kinetic energy (temperature), and cluster/nanodrop size.

It was concluded that the probability of the attacking molecule sticking to a droplet is maximal if the molecular plane is parallel or almost parallel to the droplet surface as this corresponds to multi-point interactions of relatively long n-dodecane molecules with the droplet surface. If the kinetic energy of the attacking molecules is high (greater than that corresponding to the boiling temperature) then it is expected that they will scatter and be removed from the cluster/nanodrop surface. Molecule-nanodrop interaction 
results (sticking or scattering) depend on the kinetic energy and orientations of the attacking and surface molecules. It was shown that the mechanisms of evaporation of microdrops and nanodrops are likely to involve rather different processes. In the case of microdrops, individual $\mathrm{C}_{12}$ molecules are evaporated from their surfaces, while in the case of nanodrops they can be disintegrated into clusters and individual molecules. It was shown that the decrease in the likelihood of evaporation/condensation with temperature agrees with the prediction of the classical theory based on the MD simulations of n-dodecane molecules.

The results of the estimation of the evaporation/condensation coefficient of n-dodecane molecules using the TST, based on the QC DFT approach, and taking into account the conformerisation of n-dodecane molecules, are summarised. It was shown that taking into account the QC effects leads to marginal modifications of the predicted evaporation/condensation coefficient, particularly at temperatures which are not close to the critical temperature. Thus, although the analysis of the QC effects takes into account many new processes ignored in the conventional approach, the contribution of these effects to the values of the evaporation/condensation coefficient turns out to be marginal.

\section{Acknowledgements}

The authors are grateful to EPSRC (UK) (Project EP/J006793/1) for the financial support of this project.

\section{References}

[1] S.S. Sazhin, Droplets and Sprays. Springer (2014).

[2] S.S. Sazhin, M. Al Qubeissi, R. Nasiri, V.M. Gunko, A.E. Elwardany, F. Lemoine, F., Grisch, M.R. Heikal, A multidimensional quasi-discrete model for the analysis of Diesel fuel droplet heating and evaporation, Fuel 129 (2014) $238-266$.

[3] N.A. Fuchs, Evaporation and Droplet Growth in Gaseous Media. London: Pergamon Press (1959).

[4] A.P. Kryukov, V.Yu. Levashov, S.S. Sazhin, Evaporation of Diesel fuel droplets: kinetic versus hydrodynamic models, Int. J. Heat Mass Transfer 47 (2004) 2541-2549.

[5] I.N. Shishkova, S.S. Sazhin, A numerical algorithm for kinetic modelling of evaporation processes, J. Computational Physics 218 (2006) 635-653.

[6] S.S. Sazhin, I.N. Shishkova, A.P. Kryukov, V.Yu. Levashov, M.R. Heikal, Evaporation of droplets into a background gas: kinetic modelling, Int. J. Heat Mass Transfer 50 (2007) 2675-2691.

[7] S.S. Sazhin, I.N. Shishkova, A kinetic algorithm for modelling the droplet evaporation process in the presence of heat flux and background gas, Atomization and Sprays 19 (2009) 473-489.

[8] I.N. Shishkova, S.S. Sazhin, J.-F. Xie, A solution of the Boltzmann equation in the presence of inelastic collisions, J. Computational Physics 232 (2013) 87-99.

[9] S.S. Sazhin, J.-F Xie, I.N. Shishkova, A.E. Elwardany, M.R. Heikal, A kinetic model of droplet heating and evaporation: effects of inelastic collisions and a non-unity evaporation coefficient, Int. J. Heat and Mass Transfer 56 (2013) $525-537$.

[10] C. Ledier, M. Orain, F. Grisch, J. Kashdan, G. Bruneaux, Vapour concentration measurements in biofuel sprays using innovative Planar Laser-Induced Fluorescence strategies. Proceedings of ILASS Europe 2011, 24th European Conference 
on Liquid Atomization and Spray Systems, Estoril, Portugal, September 2011 (Book of Abstracts, page 100; full paper is on the conference $\mathrm{CD})$.

[11] V.M. Gun'ko, R. Nasiri, S.S. Sazhin, F. Lemoine, F. Grisch, A quantum chemical study of the processes during the evaporation of real-life Diesel fuel droplets, Fluid Phase Equilibria, 356 (2013) 146-156.

[12] Agency for Toxic Substances and Disease Registry (ATSDR). Toxicological profile for fuel oils. Atlanta, GA: U.S. Department of Health and Human Services, Public Health Service, 1995.

[13] V.M. Gun'ko, R. Nasiri, S.S. Sazhin, A study of the evaporation and condensation of n-alkane clusters and nanodroplets using quantum chemical methods, Fluid Phase Equilibria 366 (2014) 99-107.

[14] S.S. Sazhin, I.N. Shishkova, M. Al Qubeissi, Heating and evaporation of a two-component droplet: hydrodynamic and kinetic models, Int. J. Heat and Mass Transfer 79 (2014) 704-712.

[15] B.-Y. Cao, J.-F. Xie, S.S. Sazhin, Molecular dynamics study on evaporation and condensation of n-dodecane at liquidvapour phase equilibria, J. Chemical Physics 134 (2011) 164309.

[16] J.-F. Xie, S.S. Sazhin, B.-Y. Cao, Molecular dynamics study of the processes in the vicinity of the n-dodecane vapour/liquid interface, Phys. Fluids 23 (2011) 112104.

[17] J.-F. Xie, S.S. Sazhin, B.-Y. Cao, Molecular dynamics study of condensation/evaporation and velocity distribution of n-dodecane at liquid-vapour phase equilibria, J. Therm. Sci. Tech. 7 (2012), 288-300.

[18] V.M. Gun'ko, R. Nasiri, S.S. Sazhin, Effects of the surroundings and conformerisation of n-dodecane molecules on evaporation/condensation processes, J. Chemical Physics 142 (2015) 034502.

[19] R. Nasiri, V.M. Gun'ko, S.S. Sazhin, The effects of internal molecular dynamics on the evaporation/condensation of n-dodecane, Theoretical Chemistry Accounts 134 (2015) Issue 83. DOI 10.1007/s00214-015-1681-z.

[20] P. Atkins, J. de Paula, Atkin's Physical Chemistry (Seventh Edition), Oxford: Oxford University Press (2002).

[21] D. R. Hartree, The wave mechanics of an atom with a non-Coulomb central field. Part I. Theory and methods, Mathematical Proceedings of the Cambridge Philosophical Society 24 (1928) 89-110.

[22] J.J.P. Stewart, MOPAC 2012, Stewart Computational Chemistry, Colorado Springs, CO, USA, http://openmopac.net/ (2012).

[23] J.J.P. Stewart, Optimization of parameters for semiempirical methods V: Modification of NDDO approximations and application to 70 elements, J. Mol. Modeling 13 (2007) 11731213. DOI 10.1007/s00894-012-1667-x.

[24] P.V.R. Schleyer, Editor, Encyclopedia of Computational Chemistry New York: John Wiley \& Sons (1998).

[25] P. Atkins, R. Friedman, Molecular Quantum Mechanics, Fourth edition, Oxford: Oxford University Press (2005).

[26] I.N. Levine, Quantum Chemistry, New Jersey: Pearson Prentice Hall (2009).

[27] V.M. Gun'ko, V.V. Turov, Nuclear Magnetic Resonance Studies of Interfacial Phenomena Boca Raton, New York: CRC Press, Taylor \& Francis Group (2013).

[28] A.V. Marenich, C.J. Cramer, D.G. Truhlar, Universal solvation model based on solute electron density and on a continuum model of the solvent defined by the bulk dielectric constant and atomic surface tensions, J. Phys. Chemistry 113 B (2009) 6378-6396

[29] J.J.P Stewart, L.P. Davis, L.W. Burggraf, Semi-empirical calculations of molecular trajectories: method and applications to some simple molecular systems, J. Computational Chemistry 8 (1987) 1117-1123.

[30] I.K. Ortega, O. Kupiainen, T. Kurtén, T. Olenius, O. Wilkman, M.J. McGrath, V. Loukonen, H. Vehkamäki, From quantum chemical formation free energies to evaporation rates, Atmospheric Chemistry and Physics 12 (2012) $225-235$.

[31] O. Kupiainen, I.K. Ortega, T. Kurtén, H. Vehkamäki, Amine substitution into sulfuric acid ammonia clusters, Atmospheric Chemistry and Physics 12 (2012) 3591-3599.

[32] S. Chapman, T.G. Cowling, The Mathematical Theory of Nonuniform Gases, Cambridge University Press, Cambridge (1970). 
[33] J. Zheng, S.L. Mielke, K.L. Clarkson, D.G. Truhlar, MSTor: A program for calculating partition functions, free energies, enthalpies, entropies, and heat capacities of complex molecules including torsional anharmonicity, Comput. Phys. Commun. 183 (2012) 1803-1812.

[34] J. Zheng, R. Meana-Pañeda, D. G. Truhlar, MSTor version 2013: A new version of the computer code for the multistructural torsional anharmonicity, now with a coupled torsional potential, Comput. Phys. Commun. 184 (2013) $2032-2033$.

[35] A. Lotfi, J. Vrabec, and J. Fischer, Evaporation from a free liquid surface, Int. J. Heat Mass Transfer 73 (2014) $303-317$.

[36] H. Mizuguchi, G. Nagayama, and T. Tsuruta, Seventh International Conference on Flow Dynamics (Tohoku University Press, Sendai, Japan, 2010), p. 386.

\section{Figure Captions}

Fig. 1 Interaction of an n-dodecane molecule (hot, temperature $\sim 1100 \mathrm{~K}$ ) with a cluster of seven ndodecane molecules (initial temperature $473 \mathrm{~K}$; it increases due to the interaction with a hot molecule) at the angles of attack $\Theta \approx(\mathrm{a}) 1^{\circ}$, (b) $60^{\circ}$ and (c) $90^{\circ}$. The results were obtained using the DFT B3LYP.

Fig. 2 Changes in the kinetic energy of the attacking n-dodecane molecule during its interaction with a nanodroplet of $64 \mathrm{n}$-dodecane molecules at various angles of attack: (a) $5^{\circ}$, (b) $40^{\circ}$, and (c) $45^{\circ}$, and two initial kinetic energies: (a, b) 125.5, (c) $1602 \mathrm{~kJ} / \mathrm{mol}$ (these kinetic energies correspond to the energies of both translational motion and thermal vibrations or rotations). The results were obtained using the PM7 method.

Fig. 3 Comparison of the values of the evaporation coefficient $\beta$, predicted by MD FF (symbols 1-4, curves 5-8) and Expression (6) (curve 9), versus normalised temperature $\left(T / T_{c}\right.$, where $T_{c}$ is the critical temperature). Symbols (1-4) refer to the models for structureless LJ fluids with various input parameters $[35,36]$, curves 5 and 7 refer to the results obtained based on the United Atom Model reported in [15, 16], respectively, curve 6 refers to the results of calculations based on the TST model reproduced from [15], curve 8 is based on the results of calculations using the model described by Mizuguchi et al. [30]. QC calculations were performed using DFT $\omega$ B97X-D/cc-pVTZ and SMD/ $\omega$ B97X-D/cc-pVTZ.

\section{Table Captions}

Table 1 Sums of electronic and thermal Gibbs free energies in Hartrees (1 Ha $=627.5 \mathrm{kcal} / \mathrm{mol})$ for n-dodecane conformers in the gas ( $\omega$ B97X-D/cc-pVTZ) and liquid (SMD/ $\omega$ B97X-D/cc-pVTZ) phases. 
(a) Attack angle $\Theta \approx 1^{0}$ Cluster at $473 \mathrm{~K}$ Hot molecule $x^{2}+x^{2}+y^{2}+x^{2}+y^{2}$ सt? inititis

Cluster heating to $633 \mathrm{~K}$ due to

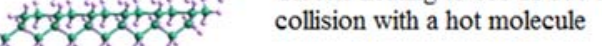

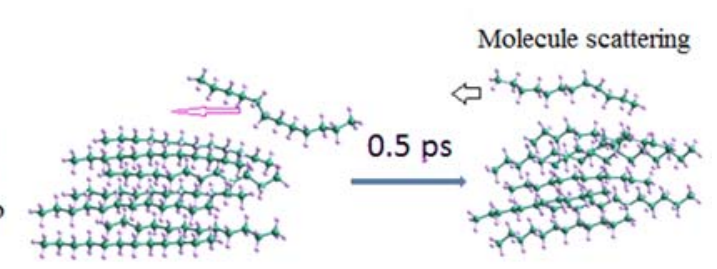

(b)
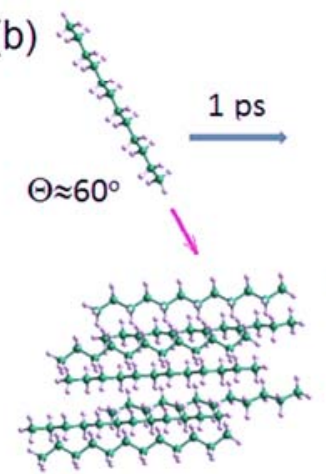

Molecule accomodation

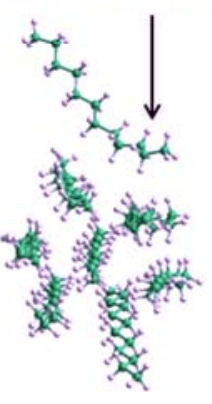

(c)

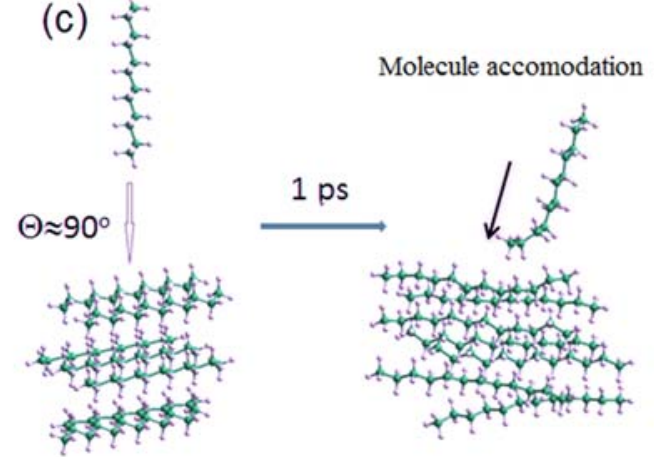

Fig. 1 

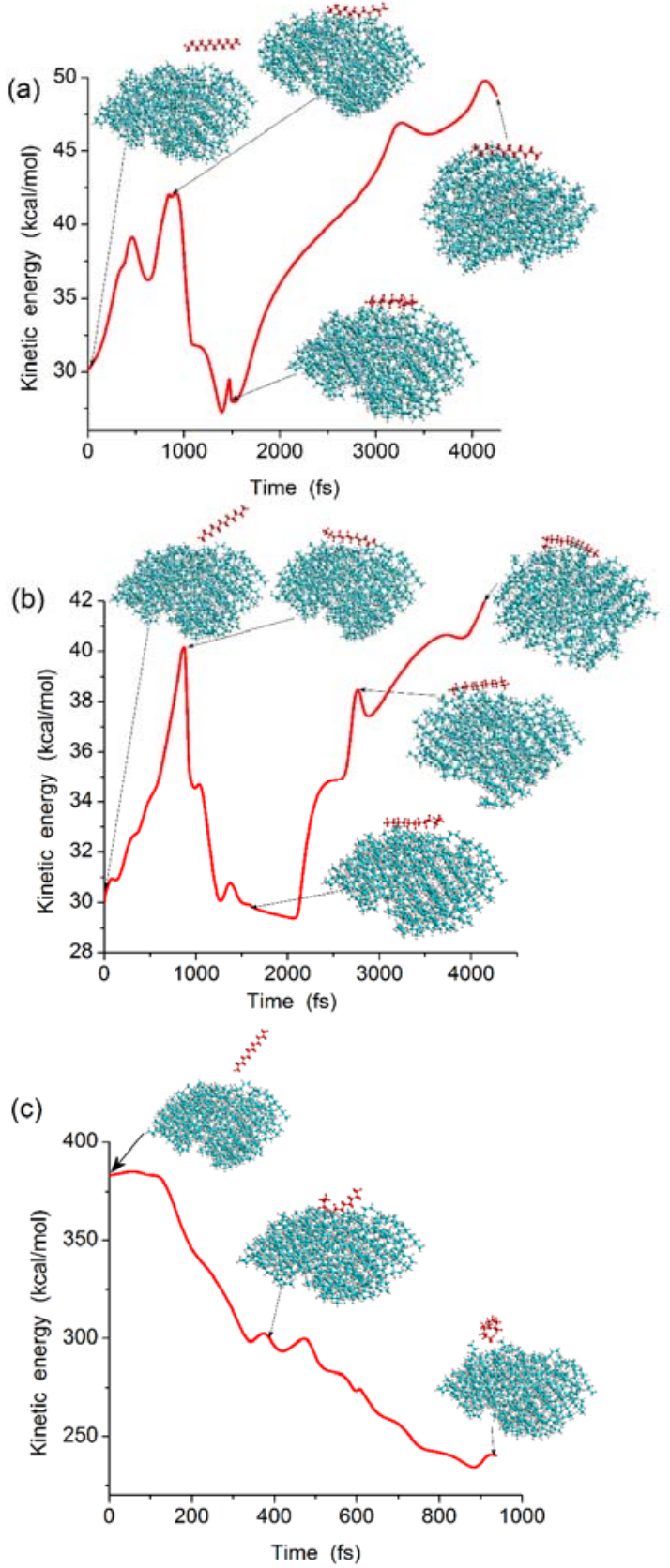

Fig. 2 


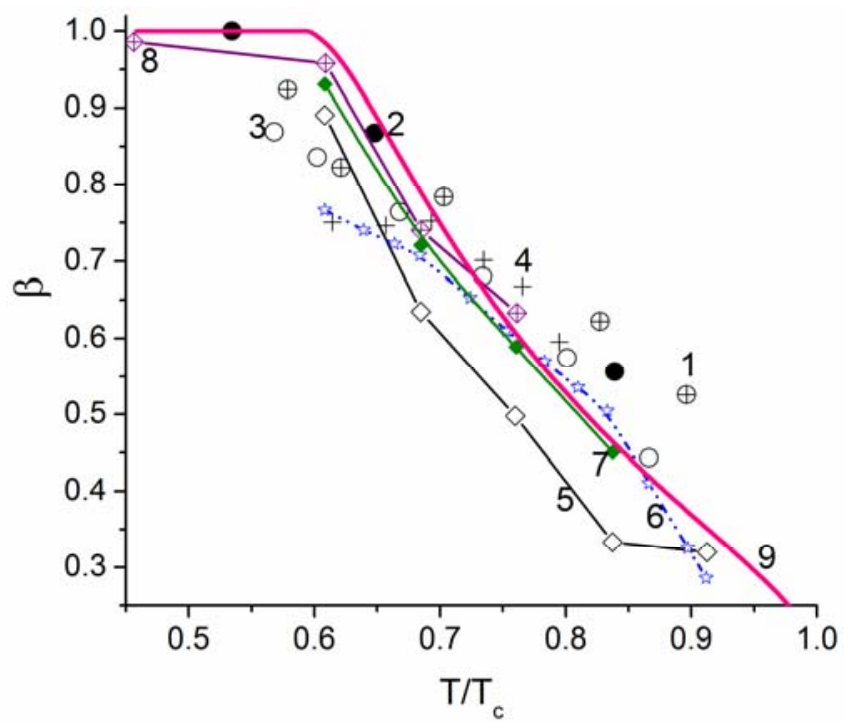

Fig. 3 


\begin{tabular}{|c|c|c|c|c|c|c|c|c|}
\hline Conformer & $\begin{array}{l}\text { Free energy, } \\
\text { gas }(\mathrm{Ha})\end{array}$ & $\begin{array}{l}\text { Free energy, } \\
\text { liquid (Ha) }\end{array}$ & Conformer & $\begin{array}{l}\text { Free energy, } \\
\text { gas }(\mathrm{Ha})\end{array}$ & $\begin{array}{l}\text { Free energy, } \\
\text { liquid (Ha) }\end{array}$ & Conformer & $\begin{array}{l}\text { Free energy, } \\
\text { gas }(\mathrm{Ha})\end{array}$ & $\begin{array}{l}\text { Free energy, } \\
\text { liquid (Ha) }\end{array}$ \\
\hline 1 & -472.672861 & -472.683897 & 33 & -472.666819 & -472.677786 & 65 & -472.668277 & -472.679477 \\
\hline 2 & -472.671955 & -472.683668 & 34 & -472.667748 & -472.678665 & 66 & -472.670404 & -472.681027 \\
\hline 3 & -472.673521 & -472.684762 & 35 & -472.675919 & -472.683376 & 67 & -472.670459 & -472.679261 \\
\hline 4 & -472.669991 & -472.681439 & 36 & -472.671182 & -472.682571 & 68 & -472.668546 & -472.679797 \\
\hline 5 & -472.673067 & -472.685107 & 37 & -472.671964 & -472.681410 & 69 & -472.670770 & -472.679867 \\
\hline 6 & -472.671544 & -472.683589 & 38 & -472.668438 & -472.679998 & 70 & -472.669831 & -472.681039 \\
\hline 7 & -472.672687 & -472.680659 & 39 & -472.671322 & -472.682050 & 71 & -472.666341 & -472.677079 \\
\hline 8 & -472.672774 & -472.684828 & 40 & -472.669002 & -472.680389 & 72 & -472.669850 & -472.680589 \\
\hline 9 & -472.673212 & -472.686184 & 41 & -472.671980 & -472.683730 & 73 & -472.667851 & -472.669995 \\
\hline 10 & -472.669906 & -472.681049 & 42 & -472.670167 & -472.679637 & 74 & -472.668430 & -472.679420 \\
\hline 11 & -472.669804 & -472.679133 & 43 & -472.673422 & -472.680413 & 75 & -472.666560 & -472.677692 \\
\hline 12 & -472.675212 & -472.685893 & 44 & -472.672426 & -472.680146 & 76 & -472.666759 & -472.678866 \\
\hline 13 & -472.672504 & -472.681944 & 45 & -472.671954 & -472.682834 & 77 & -472.667940 & -472.679737 \\
\hline 14 & -472.671916 & -472.683465 & 46 & -472.669203 & -472.679901 & 78 & -472.667880 & -472.678390 \\
\hline 15 & -472.671710 & -472.682647 & 47 & -472.669624 & -472.677674 & 79 & -472.668777 & -472.680167 \\
\hline 16 & -472.670279 & -472.679715 & 48 & -472.667998 & -472.678862 & 80 & -472.666489 & -472.677570 \\
\hline 18 & -472.667835 & -472.678967 & 50 & -472.673155 & -472.683309 & 82 & -472.667478 & -472.677968 \\
\hline 19 & -472.669889 & -472.680603 & 51 & -472.668302 & -472.679393 & 83 & -472.666826 & -472.677891 \\
\hline 20 & -472.671695 & -472.684512 & 52 & -472.666487 & -472.677630 & 84 & -472.663619 & -472.674752 \\
\hline 21 & -472.672225 & -472.683415 & 53 & -472.668599 & -472.680286 & 85 & -472.668170 & -472.677190 \\
\hline 22 & -472.668706 & -472.679769 & 54 & -472.670937 & -472.683122 & 86 & -472.664836 & -472.676118 \\
\hline 23 & -472.671085 & -472.682521 & 55 & -472.670226 & -472.677905 & 87 & -472.667376 & -472.678708 \\
\hline 24 & -472.669610 & -472.680499 & 56 & -472.671101 & -472.680866 & 88 & -472.667298 & -472.678691 \\
\hline 25 & -472.667748 & -472.678665 & 57 & -472.683570 & -472.671654 & 89 & -472.666980 & -472.678400 \\
\hline 26 & -472.670753 & -472.679134 & 58 & -472.672047 & -472.682414 & 90 & -472.664286 & -472.674884 \\
\hline 27 & -472.668744 & -472.681313 & 59 & -472.670564 & -472.681625 & 91 & -472.665538 & -472.676583 \\
\hline 28 & -472.669767 & -472.681307 & 60 & -472.670052 & -472.681207 & 92 & -472.671255 & -472.683714 \\
\hline 29 & -472.666725 & -472.677911 & 61 & -472.672385 & -472.684404 & 93 & -472.671323 & -472.682050 \\
\hline 30 & -472.670599 & -472.677483 & 62 & -472.669508 & -472.679963 & 94 & -472.664719 & -472.675590 \\
\hline 31 & -472.669418 & -472.680500 & 63 & -472.670850 & -472.681636 & 95 & -472.666980 & -472.678400 \\
\hline 32 & -472.664644 & -472.676310 & 64 & -472.668540 & -472.679033 & & & \\
\hline
\end{tabular}

